\title{
POUR UNE APPROCHE PLUS SIMPLE ET MOTIVANTE DE LA GRAMMAIRE
}

Les nombreux retours de collègues enseignants selon lesquels il devient de plus en plus difficile d'enseigner la grammaire, dans notre cas la grammaire française, soulèvent naturellement la question de savoir

- comment rendre l'enseignement des langues efficace,

- si l'enseignement de la grammaire a encore du sens et sous quelle forme,

- si on peut « optimiser les processus d'acquisition» (Thurmair 2010: 357), par lesquels les apprenants acquièrent des connaissances et des compétences grammaticales,

- comment aider les apprenants à comprendre la grammaire et à acquérir des connaissances grammaticales tout en les motivant à apprendre la langue.

Ce n'est certes pas une tâche facile pour les enseignants, mais un beau défi à relever ! Comme on le sait, « ist der Stellenwert von Grammatik im Fremdsprachenunterricht ... seit undenklichen Zeiten das beliebteste Streitobjekt der Praktiker, Didaktiker und Linguisten $»^{1}$ (Rall 2001: 880, dans: Turmair 2010: 357).

Les adeptes des approches traditionnelles partent du principe qu'en apprenant une langue étrangère, il faut à tout prix acquérir tout particulièrement la connaissance de la grammaire, qui sera ensuite automatisée dans la maitrise de la langue. L'expérience montre que cela ne fonctionne pas toujours ou seulement de manière très hésitante et qu'il y a un grand écart entre l'introduction d'une règle grammaticale et sa maîtrise réelle.

Les méthodes audio-orales, quant à elles, inspirées par le behaviourisme, affirment que la connaissance de la grammaire est insignifiante, sinon obstructive, dans la maitrise de la langue et que, par contre, l'apprentissage de la langue est plus efficace si on passe par la répétition de schémas d'énoncés qui, dans leur relation (intelligemment présentée) les uns aux autres, dans le contexte linguistique, montrent implicitement des

1 Traduction: « l'importance de la grammaire dans l'enseignement des langues étrangères... est depuis toujours la pomme de discorde parmi les praticiens, les didacticiens et les linguistes » 
régularités grammaticales. En reconnaissant implicitement le fonctionnement grammatical, les apprenants automatiseraient les traits linguistiques comportementaux liés au schéma (Portmann-Tselikas 2011: 73). Et, au plus tard depuis cette approche, la question de savoir comment les langues peuvent être acquises et quel rôle l'enseignement de la grammaire y joue, ou devrait $\mathrm{y}$ jouer, est un des thèmes centraux de la didactique des langues étrangères.

Les curriculums de l'enseignement secondaire en Autriche, par exemple, préconisent l'enseignement du vocabulaire et de la grammaire sous diverses formes contextualisées et en réseaux. Il faut enseigner le vocabulaire autant que possible dans des collocations, des expressions idiomatiques et des expressions à grammaire implicite. Les structures grammaticales quant à elles devraient être transmises sans formulation de règles en tant qu'unités lexicales - dans la mesure du possible.

Du point de vue des neurosciences et de la didactique des langues, cette recommandation de mise en réseau a un sens, notamment parce que le modèle du « lexique mental » ressemble à un gigantesque réseau dans lequel les acquisitions sont traitées et conservées.

Les découvertes en neurosciences pourraient donc constituer une aide non négligeable tant dans l'enseignement que dans l'acquisition de moyens linguistiques et de structures grammaticales, à laquelle la didactique et, en partie, les chercheurs, notamment en neurosciences ont fait référence à plusieurs reprises ces dernières années ${ }^{2}$.

\section{$2 \quad$ LES NEUROSCIENCES - UNE VOIE À SUIVRE ?}

Depuis le dernier débat suite au rapport PISA et les critiques sur la formation des enseignants qui en ont découlé, la recherche sur le cerveau a reçu un nouveau statut dans le domaine de la didactique et de la pédagogie. Des disciplines telles que la neuro-éducation ou la neuro-didactique semblaient apporter des solutions intéressantes pour la transmission des contenus d'apprentissage, la recherche sur le cerveau en tant que « discipline scientifique efficace pour le public » (traduit d'après Gröppel 2014: 176) s'est étonnamment vite transformée en une « super discipline à responsabilité universelle » (ibid.). Il faut tout de même reconnaître que même les grands spécialistes en neurologie ont souligné que cette nouvelle « leading science » (ibid. : 150) n'est pas un remède pour un enseignement efficace, mais peut contribuer à une meilleure compréhension « warum das funktioniert, was ein guter Pädagoge tut, und das nicht, was ein schlechter tut » (Roth 2009: 58). ${ }^{3}$

Il convient cependant de nuancer et d'ajouter que, après le premier buzz relatif aux découvertes faites dans la recherche sur le cerveau et leurs effets sur les processus d'apprentissage et de mémorisation, des critiques se font entendre ces dernières années et

2 cf. Roth 2009, Wewetzer 2011, Huc/Smith 2008, Macedonia 2011.

3 Traduction: pourquoi ce que fait un bon pédagogue fonctionne et ce que fait un mauvais pédagogue ne fonctionne pas. 
prennent leurs distances par rapport aux approches neurodidactiques qui croient trouver la solution à tous les problèmes scolaires dans la recherche sur le cerveau.

La critique de Göppel ${ }^{4}$ selon laquelle les représentants des neurosciences à orientation pédagogique, malgré leur grande popularité en pédagogie, n'ont pas vraiment été capables de fournir aux enseignants des concepts nouveaux, fructueux ou clarifiants (cf. ibid.: 195), est certainement justifiée, mais il faut néanmoins reconnaître (comme Göppel finit par le faire) que les recherches neuroscientifiques ont apporté des résultats précieux et passionnants pour la pédagogie et qui pourraient - en coopération avec des pédagogues et des didacticiens - rendre l'enseignement plus efficace.

La grammaire et les processus mentaux sont en soi deux domaines qui, dans la théorie récente du langage, ont donné lieu à des « arguments théoriques jusqu'au déni conscient de la pertinence d'un tel lien ». Cela s'explique finalement aussi par le fait que, pour beaucoup, les deux domaines relèvent « de la compétence de deux disciplines scientifiques différentes, à savoir la linguistique et la psychologie » (cf. Rehbein / Redder 1999: 1sq.) Mais cela n'a pas toujours été le cas.

L'interaction entre les processus mentaux et les connaissances grammaticales n'est rien de nouveau en soi. Dès le début du XIX ${ }^{\mathrm{e}}$ siècle, Wilhelm von Humboldt a reconnu le « lien entre les formes linguistiques et les formes de pensée et de culture » comme une caractéristique centrale de la langue. Il a même établi un lien entre une « analyse des formes linguistiques et une analyse de la pensée ».

Redder et Rehbein donnent un aperçu historique très intéressant quant au développement des interactions entre la grammaire et les processus mentaux de Humboldt, Steinthal, Weisgerber, de Saussure jusqu'au psychologue Bühler, qui a tenté de créer une synthèse entre le psychique et le linguistique (cf. ibid.).

Alors, pour répondre aux exigences d'un enseignement efficace, comme le préconise entre autres le curriculum de l'enseignement secondaire en Autriche, les questions suivantes se posent :

- dans quelle mesure les structures grammaticales et le vocabulaire peuvent-ils et doivent-ils être enseignés simultanément;

- comment peut-on augmenter la quantité et la qualité de cette interconnexion ;

- $\quad$ quelles stratégies d'apprentissage peuvent-elles être appliquées afin de stabiliser les réseaux neuronaux ainsi créés, pour que les acquis puissent être transférés et consolidés, et enfin, la question fondamentale :

- $\quad$ quelle « quantité de grammaire » doit (encore) être apprise ?

Nous allons essayer d'apporter une réponse à ces questions dans ce qui suit et de les illustrer d'exemples en vue d'éventuelles solutions.

4 Voir Göppel 2014, qui reflète entre autres de manière critique les approches des « Big Five », des neuroscientifiques actifs dans l'enseignement en Allemagne, Roth, Singer, Hüther, Spitzer et Bauer. 


\subsection{Vers une « économie » de l'enseignement des langues étrangères...}

L'une des découvertes les plus importantes des neuroscientifiques est certainement le fait que l'information linguistique est traitée dans des réseaux neuronaux et non - comme on le supposait auparavant - dans des «modules sensoriels isolés (vision, audition, etc.) ». Le langage n'est donc plus un phénomène abstrait de cognition, mais consiste en des connexions neuronales profondes qui combinent la vue, l'ouïe, la parole et le mouvement en unités fonctionnelles (cf. Macedonia 2011: 88).

« Brain-based education is learning in accordance with the way the brain is naturally designed to learn » (Jensen 2008: 4). Considérant que le travail du vocabulaire active à la fois l'hémisphère droit et l'hémisphère gauche du cerveau, mais que la grammaire se déroule principalement dans l'hémisphère gauche, il est logique de relier les deux et, comme l'appelle Jensen (ibid.: 22), d'activer l'apprentissage du cerveau entier, « the whole-brain-learning ». Comme on le sait, nos deux hémisphères cérébraux traitent l'information différemment, alors que l'hémisphère gauche est responsable du langage, l'hémisphère droit collecte l'information par l'image, mais ils travaillent ensemble dans le processus d'apprentissage. Sousa cite un exemple très simple, mais éloquent. Tout comme nous attrapons mieux les balles avec les deux mains (cf. Sousa 2006: 57sqq.), nous pouvons aussi recueillir et traiter plus d'informations si nous activons nos deux hémisphères. L'acquisition via différents canaux de perception, la répétition constante et la consolidation de certains schémas neuronaux favorisent la mise en réseaux des savoirs. Il s'agit donc d'une approche multidisciplinaire qui vise à favoriser l'inclusion de la nature du cerveau dans la prise de décision. « Brain-based approach », l'approche fondée sur le cerveau n'est pas encore en soi une recette pour un bon enseignement. Mais si nous réfléchissons à ce que nous savons déjà sur le cerveau, alors enseigner et apprendre « with the brain in mind », avec le cerveau en tête (cf. ibid.), nous pourrions prendre des décisions plus efficaces et atteindre plus d'apprenants, mieux et plus souvent. Les neurosciences ont donné des résultats utiles et convaincants à cet égard, qui peuvent à leur tour contribuer à la réussite de la conception des leçons, à de meilleurs résultats d'apprentissage et aussi à une augmentation de la motivation des apprenants s'ils sont utilisés à bon escient. Parce que « das lernende Gehirn hält verschiedene Lernmöglichkeiten bereit ${ }^{5}$ ( $\mathrm{cf}$. Brand / Markowitsch 2009: 81).

\section{2 «Moins, c'est souvent plus »}

Réussir un réseau nécessite deux choses : la quantité et la qualité. La première est évidente - plus j'utilise des mots, une collocation ou même une forme verbale, plus je les

5 « le cerveau en mode d'apprentissage offre diverses façons d'apprendre » 
retiens vite. Pour une qualité optimale, Brand et Markowitsch (2009: 81) défendent la thèse selon laquelle les apprenants obtiennent de meilleures performances d'apprentissage et surtout de rétention si l'on tient compte de la « réduction des besoins en mémoire et d'une stratégie spécifique pour l'augmentation ciblée de certains apprentissages et performances de mémoire ». À ne pas oublier, bien sûr, d'autres compétences cérébrales comme l'attention, la concentration et surtout la motivation.

Mais comment réduire les exigences imposées à la mémoire selon la devise « moins, c'est souvent plus » lors de l'apprentissage d'une langue étrangère ?

Grâce à un aperçu global au début de la leçon, suivi d'instructions « étape par étape » . Le programme d'études et le plan d'apprentissage sont présentés sur une grande image, suivis des détails qui sont présentés en sous-titres. Par la suite, il y aura des alternances répétées entre la grande image et les sous-titres (cf. Jensen 2008: 22). Ainsi, les apprenants sont préparés à ce qu'ils apprennent par " priming » (traitement inconscient, ce qui à son tour conduit à une meilleure performance de reconnaissance). De plus, cette structuration et cette organisation de l'unité d'enseignement servant comme « aide au stockage externe », ces éléments peuvent ensuite être utilisés à nouveau comme une incitation à rappeler ce qui a été appris (Brand /Markowitsch 2009: 82).

Dominique Abry et Marie-Laure Chalaron (1996: 4) dans leur livre « La grammaire des premiers temps, volume 1 » fournissent un exemple très réussi de réduction de la grammaire dans les premières leçons. Elles s'appuient sur Dubois et sa grammaire structurelle et plaident pour une classification des verbes au présent selon les régularités phonétiques. C'est ainsi que les verbes sont appris dans les leçons initiales selon leurs terminaisons identiques et non selon le schéma traditionnel «1er, 2e, 3e - groupe » .

L'exemple des verbes en -er (à 1 base) au présent illustrera leur catégorisation par classement phonétique:

Verbes réguliers en -er avec l'indice que ces verbes représentent $90 \%$ des verbes français :

\begin{tabular}{|l|l|l|}
\hline 1. Je & parl & e \\
\hline 2. Tu & parl & es \\
\hline 3. Il/Elle/On & parl & $\boldsymbol{e}$ \\
\hline 6. Ils/Elles & parl & ent \\
\hline 4. Nous & parl & ons \\
\hline 5. Vous & parl & ez \\
\hline
\end{tabular}

L'apprentissage des verbes au présent en - er se concentre ici sur la régularité phonémique des trois premières personnes au singulier et de la troisième personne au pluriel. En éliminant les explications compliquées, les exceptions, les terminaisons plurielles, ainsi qu'au début le pluriel à la 1ère et 2ème personne, et en introduisant les applications 
répétées à d'autres exemples tels que aimer, écouter, habiter, les terminaisons phonétiques mais aussi graphiques sont automatisées et pourraient être plus facilement réappliquées en apprenant de nouveaux verbes. Considérant que tous les verbes français - à l'exception de être, avoir, aller et faire - ont la même régularité phonétique au singulier, cette réduction et cette concentration sur la prononciation des verbes dans les leçons initiales contribuent fondamentalement au fait que les conjugaisons s'apprennent beaucoup plus facilement ultérieurement, comme de nombreuses expériences avec mes apprenants le montrent. Le fait que les terminaisons soient écrites différemment est alors automatiquement mémorisé.

Les verbes ne sont donc pas seulement stockés selon leur signification, mais aussi selon les formes phonétique, graphémique, morphologique, syntaxique et sémantique. Dans la forme phonétique, par exemple, on a constaté que certaines parties du mot sont stockées plus profondément, ce qu'Aitchison appelle « Badewanneneffekt», (Merz 2009: 3), l'effet baignoire. C'est à dire que les sons au début et à la fin du mot sont stockés de manière plus accessible que le reste du mot, comme si le mot était une personne étendue dans une baignoire, avec tête et pieds à chacune des extrémités.

Bien sûr, ces connaissances phonologiques ne suffisent pas à elles seules. La connaissance syntaxique, qui définit la position du mot dans la phrase, peut également être optimisée en ajoutant des structures grammaticales simples telles que « je parle français, il parle allemand, ils parlent espagnol, etc. ». Ceci active simultanément les connaissances morphologiques, structurelles et sémantiques.

L'approche d'Abry est très intéressante dans la mesure où elle lie les règles grammaticales aux particularités phonétiques, ce qui est malheureusement trop souvent négligé dans les cours de français, comme le souligne également Berri :

«Une bonne compétence communicationnelle n'est pas seulement comme le pensent généralement de trop nombreux didacticiens, une maitrise convenable de la syntaxe et du lexique assez conséquent. C'est aussi, comme le précise Pagel (1996) une maîtrise des aspects phonétiques et, en premier lieu, des éléments prosodiques » (Berri 2007: 247).

La sémantique, et donc aussi la grammaire, sont étroitement liées à la phonétique, puisque tous les sons que nous produisons pour communiquer veulent transmettre un sens. Produire correctement des sons signifie aussi produire un sens. Conjuguer correctement des verbes, mais surtout les prononcer correctement, c'est aussi transmettre leur sens et se faire comprendre, parce que - comme le souligne à juste titre Berri - sans une bonne prononciation orale, nous n'avons aucune possibilité de transmettre un message qui soit vraiment et correctement compris, même si les mots étaient bien choisis et la syntaxe correcte (voir ibid.). Il suggère donc de changer l'ordre des niveaux d'acquisition des langues, car si l'on veut être compris, il faut « avant tout être capable de produire des suites phonétiques correctes » (ibid.: 248).

Pour cette raison, de nombreux didacticiens soutiennent que l'apprentissage des langues étrangères devrait d'abord commencer par la langue orale et puis être suivie par la partie écrite - un aspect qui sera abordé plus en détail dans un chapitre ultérieur. 
Une bonne prononciation et une bonne compréhension favorisent également la motivation de l'apprenant, abaissent les seuils d'inhibition et consolident ce qui a déjà été acquis. « La maîtrise de la prononciation, du fait même qu'elle dégage un encouragement important, mène à une acquisition des aspects grammaticaux et lexicaux de manière plus efficace, justement lorsque l'apprenant aura pris confiance en lui parce qu'il sait prononcer. Il n'hésitera pas à intervenir, c'est à dire à pratiquer la langue » (ibid.: 249).

\subsection{Comment rendre la grammaire « jolie »?}

Puisque le langage et les gestes sont très proches l'un de l'autre, peut-être même de la même origine, les gestes, ainsi que les expressions faciales, peuvent être utilisés de manière très profitable pour l'apprentissage du vocabulaire ou des structures grammaticales, car ils soulignent encore davantage le sens. Il est bien connu que les apprenants se souviennent mieux du vocabulaire lorsqu'ils ajoutent une autre compétence. Que ce soit par l'image, le geste, l'haptique. De plus, la référence à la mélodie de la parole est toujours établie en premier lieu lorsqu'on parle d'une langue.

Les gestes et les expressions faciales sont aussi inséparablement liés à l'expression orale, ce qui accroît la mise en réseau. Les zones cérébrales correspondantes - le cortex moteur et le cortex sensoriel - sont juxtaposées et étroitement liées, puisque la même moitié du cerveau gère la valeur symbolique des mots, des gestes et des expressions faciales (Huc 2008 : 31). ${ }^{6}$

De plus, les découvertes neuroscientifiques ont montré que les caractéristiques prosodiques telles que l'intonation, l'accentuation, le rythme et la mélodie du son jouent un rôle décisif dans l'acquisition du langage. La prosodie en particulier, qui a les mêmes circuits neuronaux que la musique, est d'une grande importance, surtout dans le domaine de la syntaxe, car elle nous permet de séparer les mots d'une phrase entendue et de nous concentrer sur les informations nouvelles ou importantes de la phrase (cf. ibid.).

Pour bien comprendre le sens d'un énoncé oral, l'accentuation, le rythme des phrases et, surtout, l'intonation sont beaucoup plus décisifs que les mots utilisés. Il est très difficile de comprendre une phrase mal accentuée (Parizet 2008: 116). Bien sûr, le langage écrit accentue aussi la séparation des mots, mais notre cerveau semble beaucoup plus réceptif au langage parlé, tout comme nous sommes beaucoup plus réceptifs à la musique qu'aux partitions.

Par conséquent, les apprenants devraient être sensibilisés à certaines caractéristiques prosodiques du français le plus tôt possible.

6 Concernant la communication non verbale d'un point de vue didactique en langue étrangère voir également Reimann 2016 
Avec son spectacle « Ritmimots " ${ }^{7}$, Régine Llorca a mis en scène l'interaction entre le rythme, l'expression faciale et les mots avec des exemples très convaincants et a montré en même temps à quel point la mise en réseau peut aider à améliorer la prononciation et donc la compréhension de la parole, mais aussi la compréhension pour la langue elle-même. Dans son émission sur le Bayerischer Rundfunk, elle explique la rapidité avec laquelle on peut se concentrer sur la mélodie de la phrase grâce à une musique de fond rythmique.

Elle illustre sa méthodologie entre autres par la phrase d'exemple : "Approchez Mesdames Messieurs » et accentue les syllabes des mots en tapant dans les mains et en élevant la voix. L'élément gestuel renforce ainsi le rythme. Une approche familière en soi, car depuis la petite enfance « l'acquisition du langage... est soutenue par le mouvement. Cela commence par le chant et le jeu des doigts et se poursuit à la maternelle ou à l'école primaire avec des chants soutenus par des gestes et des mouvements » (Seeringer 2015: 11).

Selon Llorca, il est très facile de mettre l'accent sur les syllabes correctes, « il suffit de sentir où on bouge en parlant », car l'accent n'est rien d'autre que l'énergie qui est dans le corps. Alors elle renforce les syllabes accentuées par des mouvements de la tête, des mains ou même simplement des sourcils. En même temps, la phrase obtient sa propre mélodie, un rythme à trois mesures, semblable à une valse.

Approchez Mesdames Messieurs

Puis elle ajoute dans le même rythme : venez ! ! entrez ! entrez ! !

Chaque apprenant peut comprendre cette mélodie de la phrase par une écoute consciente, puisqu'elle existe déjà, « alors il suffit de savoir écouter la phrase, pour faire sortir sa musique ». Et une fois le bon rythme et la mélodie de la phrase trouvés, alors cette phrase résulte en un thème, une idée. Comme exercice supplémentaire, on peut demander aux apprenants de trouver d'autres thèmes qui ont le même rythme. Les phrases nouvellement formées devraient couvrir des sujets réalistes, tels que p.ex. « faire les courses au marché » :

Des pommes, des poires, et voilà de belles tomates !

L'accentuation renforce la signification de ces phrases ; on sait que quelqu'un est appelé pour lui montrer quelque chose, ou pour lui demander de faire quelque chose. L'accent mis au début a pour but d'attirer l'attention. Et c'est précisément cette intonation qui est nécessaire pour les verbes de l'impératif : approchez, venez...

La musique, dans notre cas la mélodie de la phrase, est alors intentionnellement utilisée pour faire des exercices de grammaire ou pour introduire, pratiquer et consolider un nouveau vocabulaire. Régine Llorca résume dans l'interview combien il est important de bien présenter l'ensemble, «faire joli », c'est-à-dire de motiver l'apprenant par des conditions cadres agréables.

7 L'exemple suivant est tiré de la première émission de radio scolaire (1999) d'une série en sept parties « Ritmimots : le français par l'oreille musicale » intitulée : La pulsation magique. L'interview avec Régine Llorca sur Bayern 2 Radio a été réalisée par Francine Singer. 
Elle évoque aussi la possibilité de s'amuser avec des mélodies de phrases en prononçant plusieurs phrases avec le même rythme en même temps. Elle utilise donc les techniques de « chansons...pour la parole». Par son approche multidisciplinaire, l'interaction de différentes compétences et la mise en réseau qui en résulte, elle montre et aux apprenants et aux enseignants qu'une « réorientation » de l'enseignement grammatical peut conduire à des résultats très efficaces.

La langue parlée et les gestes forment ensemble un «système de communication qui s'est développé avec succès depuis des milliers d'années » (Macedonia 2012: 89). Après tout, nous apprenons par le biais d'exemples, pas de « l'intériorisation d'une règle abstraite » (Multhaup dans : Thurmair 2010: 362). Bien sûr, cela exige des exemples convaincants, de qualité mnémotechnique et encore mieux des exercices sociométriques. Il semble efficace de montrer la prononciation des sons et de demander aux élèves de les imiter (Macedonia 2012: 91).

Les neurones miroirs sont responsables du fait que dans notre cerveau, seule l'observation d'un mouvement par un locuteur stimule les mêmes voies neuronales que si nous avions effectué les mouvements nous-mêmes (Huc / Vincent Smith 2008: 31).

Pierre Huc, neuropsychiatre, et Brigitte Vincent Smith, spécialiste en didactique, concluent dans leurs travaux de recherche communs que, surtout pour les apprenants A1, l'accent doit être mis sur les exercices oraux, qui doivent être réalisés avec les expressions faciales et les gestes, et que « les méthodes qui activent les mêmes aires cérébrales que le langage parlé spontané, en dehors de toute contrainte grammaticale ou écrite, sont donc probablement les plus efficaces » (ibid.: 31.).

Ce qui soulève bien sûr la question de savoir quelles méthodes devraient être utilisées pour, d'une part, stabiliser les réseaux neuronaux qui se sont développés et d'autre part pour transférer ce qui a été appris dans la mémoire à long terme.

\section{QUE FAIRE POUR QUE CE QUI A ÉTÉ APPRIS RESTE DANS NOTRE MÉMOIRE?}

La mémoire à long terme est pratiquement illimitée en termes de temps et de nombre d'unités de mémoire. Une différenciation bien connue est, par exemple, la division en contenus déclaratifs (que l'on peut expliquer) et non déclaratifs (Squire 1987).

Cependant, il existe également des subdivisions en « systèmes organisés hiérarchiquement », comme les quatre catégories de mémoire de Tulving (Tulving 1995) et Markowitsch (Brand/Markowitsch 2009: 71), qui en a même rajouté une cinquième:

1. la mémoire épisodique, le système « hiérarchiquement le plus élevé » qui stocke les événements qui ont une relation claire avec l'espace, le temps et les situations, comme les autobiographies ou les voyages dans le temps 
2. la mémoire sémantique, également appelée système de connaissances, qui contient par exemple des connaissances sur les formules mathématiques ou les capitales des pays

3. la mémoire perceptuelle, qui nous permet de reconnaître des objets, des personnes, des sons ou des stimuli sur la base d'un sentiment de familiarité. Tulving et Markowitsch la décrivent comme « présémantique », c'est-à-dire qu'il n'est pas nécessaire de pouvoir nommer d'où on connaît l'objet correspondant, etc.

4. le priming qui conduit à une meilleure reconnaissance par des choses déjà inconsciemment connues

5. la mémoire procédurale, qui fonctionne en grande partie inconsciemment et qui inclut les habiletés motrices comme la natation, etc. Son contenu n'est pas ou difficilement verbalisable.

Ce ne sont pas toutes les informations que nous obtenons qui entrent dans la mémoire à long terme. La sélection des stimuli, qui sont traités en premier lieu, est faite par la mémoire de temps ultra-court. Seuls les contenus d'intérêt peuvent être stockés.

Sarah-Jayne Blakemore et Uta Frith ${ }^{8}$ (2005: 152) l'illustrent par un exemple très simple : il s'agit d'examiner attentivement les lettres suivantes et d'essayer de mémoriser leur ordre en une minute.

\section{P P N Q T D D C A D J P M N O P M P S}

Sans moyens mnémotechniques, on ne se souviendra sûrement que d'une fraction, et/ou de certaines des premières lettres. Mais si on obtient de l'aide, par exemple le texte du refrain (petit papa noël, quand tu descendras du ciel avec des jouets par milliers, n'oublie pas mes petits souliers), on se replie - dans ce cas - sur la mémoire sémantique, ce qui fait à son tour rappeler plus de lettres dans leur ordre, sinon toutes.

Les contenus d'apprentissage avec un sens ou des associations activent une zone supplémentaire du cerveau, le « cortex préfrontal inférieur gauche » (ibid.).

La mémoire à court terme, une « mémoire passive» (Brand/Markowitsch 2009 : 71) ne convient que pour une répétition immédiate. Elle est également assez limitée et ne peut enregistrer qu'environ 7 informations détaillées à la fois et les stocker pendant environ 15 à 20 secondes. Les informations importantes par contre sont immédiatement enregistrées (Blakemore 2005: 152).

8 Blakemore et Frith citent l'exemple de Twinkle, petite étoile scintillante, et vu le contexte, j'ai choisi un célèbre chant de Noël français. 
COMMENT STOCKER L'ACQUIS - OU L'ASSOCIER AVEC CE QUI EST FAMILIER

Le stockage peut se faire, par exemple, par l'association de nouvelles choses à apprendre et de contenus de la mémoire à long terme. Une bonne structuration ou organisation du matériel d'apprentissage facilite ce processus. Par exemple, lors de l'apprentissage $\mathrm{du}$ vocabulaire, le regroupement en fonction du contenu ou de critères phonétiques est avantageux. Ces catégories sont ensuite d'importants conseils de récupération lors de la mémorisation ultérieure.

Dans sa thèse Kalka (2002: 29sqq.) donne un aperçu très complet des différentes possibilités et stratégies d'enseignement du vocabulaire, qui peuvent également être appliquées aux unités grammaticales. Elle traite entre autres des stratégies de pensée de Rohrer et mentionne « l'abstraction, c'est-à-dire la recherche de caractéristiques communes » . Différents vocabulaires sont ainsi donnés, ce qui permet de trouver des caractéristiques communes. La « pensée taxonomique » est une hiérarchisation de «l'information codée linguistiquement » par la superordination, la subordination et l'ordre secondaire.

Les exercices avec des verbes sont particulièrement adaptés à cette fin, surtout des verbes qui expriment une certaine procédure. Un exemple serait un exercice avec des verbes réflexifs sur le sujet " le matin »: se réveiller, se lever, se laver, se brosser les dents... Bien sûr, cet exercice pourrait aussi être soutenu par du matériel visuel, car des études empiriques ont montré que les élèves apprennent mieux des mots accompagnés d'images que des mots seuls.

Les apprenants à qui on présente des mots et des images ont l'occasion de construire des modèles mentaux et verbaux et d'établir des liens entre les deux. Cependant, si l'apprenant n'est confronté qu'aux mots, il a la possibilité de construire un « verbal mental model », mais il est peu probable qu'il construise un " pictural mental model » et établisse des liens entre les deux (Mayer 2001: 63). Bien sûr, il est préférable d'utiliser des images « figuratives » qui évoquent directement des associations et déclenchent donc des réactions « affectives » plutôt que des images purement « logiques» (Hecke 2010: 11).

Les associations aident à conserver les connaissances en reconnaissant les relations. Comme notre mémoire peut aussi très bien stocker des schémas, nous formons des analogies, c'est-à-dire que nous reconnaissons des relations de référence.

Le stockage est ensuite suivi de la phase de consolidation. Les références à tout ce qui est connu, familier, aux sujets qui ont déjà été traités, y compris les disciplines voisines, facilitent la consolidation du nouveau à l'apprenant (cf. Brand/Markowitsch 2009: 73).

Pour présenter la grammaire d'une manière efficace et simplifiée, il faudrait faire beaucoup plus de références à ce qui est familier. Prenons l'exemple de l'introduction des questions en français. Pour ce chapitre de grammaire, la comparaison avec d'autres langues s'est avérée très utile. 
Les trois types de questions français s'expliquent très bien par - dans notre cas des comparaisons avec la langue maternelle allemande ou l'anglais que les apprenants connaissent bien.

\section{Inversion :}

Français : Où vas-tu?

Allemand : Wohin gehst du?

\section{Question avec est-ce que :}

Français : Où est-ce que tu vas ?

Anglais: Where do you go?

\section{Intonation:}

Français : Tu vas où?

Allemand: Du gehst wohin? (utilisé en allemand seulement quand la fin de la phrase n'a pas été comprise)

Le processus d'apprentissage est facilité par l'association avec le familier. La compréhension grammaticale s'effectue à travers un processus cognitif en partie basé sur « la connaissance grammaticale inconsciente et construit une représentation mentale de la structure grammaticale des phrases à partir de la séquence linéaire des unités lexicales » (Fabricius - Hansen 1999: 16). Bien sûr, dans le sens d'une réduction des exigences, il ne faut pas introduire les trois types de questions en même temps, car cela peut être plutôt contre-productif et peut être source de confusion, mais l'expérience des unités d'enseignement a montré que cette approche est très positive et donne de très bons résultats.

Dans leur manuel scolaire interlingual « Découvrons le français » (Rückl et alii, 2013), Michaela Rückl et ses collègues mettent en réseau la langue à apprendre avec des connaissances linguistiques déjà acquises et connues dans d'autres langues romanes et optimisent ainsi considérablement le succès de l'apprentissage.

\section{5}

\section{METTRE L'ACCENT SUR L'ORAL}

Claude Germain de l'Université du Québec et Joan Netten de l'Université de Terre-Neuve ont présenté une étude très intéressante.

Ils divisent la grammaire pour l'acquisition d'une langue seconde et d'une langue étrangère en deux groupes : la grammaire interne, qui n'est importante que pour la production orale, et la grammaire externe pour la langue écrite, examinent les différences entre la langue parlée et écrite en utilisant l'exemple du français, et utilisent leur concept 
développé pour démontrer l'influence significative que la grammaire interne - c'est à dire la production orale - a sur la production écrite.

Ils concluent qu'une correction plus intensive de la prononciation et des erreurs d'expression orale et de production améliorera avant tout la production écrite. Des études menées dans des écoles canadiennes et chinoises auprès de centaines d'élèves ont donné des résultats prometteurs (Germain/Netten 2013: 15sqq.).

Dans leur étude, ils se réfèrent à la théorie de Michel Paradis (Paradis 2004, 2009), qui distingue entre mémoire déclarative et mémoire procédurale.

La première provient du domaine du conscient et inclut le savoir métalinguistique (vocabulaire, règles de grammaire, conjugaison des verbes), tandis que la mémoire procédurale comprend le domaine du non conscient - et inclut, par exemple, l'aptitude à communiquer. Les deux dépendent de processus cérébraux complètement différents (Germain/Netten 2013: 18).

Dans leur approche neurodidactique de la médiation linguistique, Germain et Netten font donc une distinction très stricte entre ces deux types grammaticaux. Dans ce contexte, ils soulignent également le fait que le mot grammaire vient du latin grammatica (l'art de tracer des lettres) et que les grammaires ont été inventées pour apprendre à écrire une langue qui était déjà parlée. Ils plaident donc pour l'apprentissage de deux grammaires - une interne pour la langue parlée et une externe pour la langue écrite, ainsi que la séquence d'apprentissage - langue parlée - lecture - langue écrite.

$\mathrm{Si}$ on commence seulement par le langage oral au début, les étudiants développent leur grammaire interne. Les élèves ayant un niveau de débutant ou de faux débutant écrivent très souvent comme ils le pensent spontanément, c'est-à-dire les structures linguistiques qu'ils ont déjà automatisées en langage parlé. Et si cette grammaire interne est incorrecte, les structures grammaticales de la langue écrite le seront aussi.

D'où l'importance de corriger d'abord les erreurs dans la langue parlée afin d'améliorer la compétence de la langue écrite. Pour pouvoir lire correctement, les élèves doivent être capables de faire le bon lien entre les mots qu'ils lisent et de leur donner un sens.

Mais si ces connexions n'ont pas été faites oralement au préalable, l'apprenant a tendance à lire mot à mot sans comprendre le sens réel de la phrase. Il doit aussi être capable de faire un lien correct entre le son et son écriture. Prenons l'exemple simple, mais efficace : ils ont des enfants - s'il prononce mal, il lit ils sont des enfants, ce qui a un sens tout à fait différent. Il est important d'observer d'abord comment le mot est écrit avant de l'écrire soi-même. Et c'est exactement ce que nous faisons quand nous apprenons notre langue maternelle. D'abord on imite, on parle, on nous fait la lecture et on lit inconsciemment, puis on apprend à écrire (cf. ibid.: 15 sqq.).

Mohammed Al-Khatib appelle la grammaire explicite la grammaire franche parce qu'elle ne cache pas la réalité et l'importance de la grammaire dans l'acquisition du langage. Il appelle la grammaire implicite grammaire cachée car, à son avis, elle cherche à cacher sa difficulté aux apprenants. Il soulève également la question légitime de savoir si 
la grammaire implicite ou interne ou mentale seule peut aider à l'acquisition du langage communicatif.

Cependant, certains élèves trouvent cela difficile parce qu'ils ont besoin d'un cadre grammatical sur lequel s'appuyer. Bien sûr, ils doivent être guidés par l'enseignant à la règle grammaticale (cf. Al-Khatib 2008).

Le rôle de l'enseignant est certes un rôle clé. Surtout dans l'enseignement avec des approches très communicatives, où « les structures linguistiques et la grammaire ne sont souvent pas cognitives et explicitées ", l'enseignant doit avoir une connaissance grammaticale très solide, comme le dit fort justement Maria Thurmair (Thurmair 2010: 360).

Il devrait avant tout promouvoir la « language awareness », la conscience linguistique des élèves et avoir des connaissances approfondies en termes de fréquence, de fiabilité, de portée, d'exceptions et être capable d'évaluer correctement le niveau de difficulté (cf. ibid.: 361). Un beau défi.

\section{CONCLUSION}

Une distinction entre la grammaire interne, qui n'est importante que pour la production orale, et la grammaire externe pour la langue écrite dans les cours pour débutants, surtout en français, semble pertinente, d'autant plus qu'en réduisant la grammaire à l'essentiel, en se concentrant sur l'expression orale et la prosodie, et en mettant en réseau différents canaux de perception, on parvient à une immersion plus intensive dans la nouvelle langue et, en même temps, à renouer avec les expériences antérieures réussies, ce qui a pour effet de motiver les élèves pour la langue étrangère.

Parce que chaque apprenant veut pouvoir communiquer le plus rapidement possible. Après cette phase initiale, cependant, il ne faut pas se passer d'explications grammaticales explicites, car la compréhension de la terminologie de base et la connaissance du travail grammatical constituent une «boîte à outils de base » pour l'apprentissage des langues tout au long de la vie (Fayndrich 2000: 7) et favorisent aussi l'autonomie de l'apprenant.

Cependant, les cours de grammaire devraient toujours être mis en réseau et intégrés dans une variété de méthodes. Les découvertes neuroscientifiques n'apportent pas de solutions en soi, mais des suggestions intéressantes pour une réorientation de l'enseignement de la grammaire. Et par la suite peut-être une (re-)motivation des élèves pour la langue de Molière... 


\section{BIBLIOGRAPHIE}

AL-KHATIB, Mohammed (2008) Faut-il ignorer complètement la grammaire explicite en FLE ? En ligne, le 29/1/2016. http:/gramm-r.ulb.ac.be/fichiers/colloques/ Nantes2008/ALKHATIB.pdf.

BERRI, André (2007) Quelques considérations à propos de la phonétique dans la formation des futurs enseignants de FLE. Fragmentos 33, 245-50.

BLAKEMORE, Sarah-Jayne/Uta FRITH (2005) The Learning Brain. Lessons for Education. Malden, États-Unis : Blackwell Publishing.

BRAND, Matthias/Hans J. MARKOWITSCH (2009) Lernen und Gedächtnis aus neurowissenschaftlicher Perspektive - Konsequenzen für die Gestaltung des Schulunterrichts. U. Hermann (dir.), Neurodidaktik. Grundlagen und Vorschläge für gehirngerechtes Lehren und Lernen. Weinheim et Bâle : Beltz, 69-86.

FABRICIUS-HANSEN, Cathrine (1999) Grammatik und Verstehen. A. Redder et J. Rehbein (dir.), Grammatik und mentale Prozesse. Tübingen : Stauffenbergverlag, 15-31.

FAYNDRICH, Christian (2000) Ist der kommunikative Ansatz im Fremdsprachenunterricht an seine Grenzen gekommen? German Studies at Aston 1, 2-12.

GEAKE, John G. (2009) The Brain at School. Educational Neuroscience in the Classroom. Maidenhead, Angleterre : MCGrawHill Open University Press.

HABERMANN, Mechthild (dir.) (2010) Grammatik wozu? Vom Nutzen des Grammatikwissens in Alltag und Schule. (Thema Deutsch, 11). Mannheim : Dudenverlag.

GERMAIN, Claude/Joan NETTEN (2013) Grammaire de l'oral et grammaire de l'écrit dans l'approche neurolinguistique (ANL). Synergies Mexique 3, 15-29.

GÖPPEL, Rolf (2014) Gehirn, Psyche, Bildung. Stuttgart: W. Kohlhammer.

HECKE, Carola/Carola SURKAMP (2010) Bilder im Fremdsprachenunterricht. Neue Ansätze, Kompetenzen und Methoden. Tübingen : Narr.

HEINE, Lena (2014) Unterrichtsbezogene Fremdsprachenerwerbstheorien und neurowissenschaftliche Erkenntnis. H. Böttger et G. Gien (dir.), The multilingual brain. Zum neurodidaktischen Umgang mit Mehrsprachigkeit. Eichstätt : Epubli.

HERRMANN, Ulrich (dir.) (2009) Neurodidaktik. Weinheim et Bâle : Beltz.

HUC, Pierre/Brigitte VINCENT SMITH (2008) Naissance de la neurodidactique. Le français dans le monde 357, 30-31.

JENSEN, Eric (2001) Different Brains. Different Learners. Thousand Oaks, Californie : Corwin Press.

KALKA, Katarzyna (2002) Effizienz verschiedener Lehrmethoden zur Wortschatzvermittlung. Marburg : Tectum Verlag.

KOCH, Corinna (2015) Dienen, nicht dominieren. Der fremdsprachliche Unterricht Französisch. Grammatik umdenken 135, 2-9. 
KÖPCKE, Klaus-Michael/Arne ZIEGLER (dir.) (2011) Grammatik - Lehren, Lernen, Verstehen. Berlin : De Gruyter.

JENSEN, Eric (2008) Brainbased Learning. The New Paradigm of Teaching. Thousand Oaks, Californie : Corwin Press.

LANGE, Ulrike C. (2015) Französisch ist schick und schlau! Für die offensive Verwendung einer Weltsprache. Französisch heute 2, 43-44.

MACEDONIA, Manuela (2012) Wie konkret kann die Hirnforschung Pädagogen helfen? En ligne, le 16/10/2019. http://www.macedonia.at/wp-content/uploads/2012/10/ Macedonia_Lerntheorien_10.pdf.

MAYER, Richard E. (2001) Multimedia Learning. Cambridge : University Press.

MÜLLER, Thomas (2016) Anspruch und Scheitern einer Verbindung von Neurowissenschaften und Didaktik. R. Porsch (dir.), Einführung in die Allgemeine Didaktik: ein Lehr-und Arbeitsbuch für Lehramtsstudierende. Münster, New York : Waxmann, 207-229.

PARADIS, Michel (2004) A neurolinguistic theory of bilingualism. Amsterdam/Philadelphie : John Benjamins.

PARADIS, Michel (2009) Declarative and Procedural Determinants of Second Languages. Amsterdam/Philadelphie : John Benjamins.

PARIZET, Marie-Louise (2008) Phonétique et Cadre Commun: propositions pour un cours de FLE. Synergies Espagne 1, 113-122.

MERZ Guiliano/Julian PAGET/Andreas PETÖ (2009) Das mentale Lexikon und die intuitive Grammatik. En ligne, le 16/10/2019. http:/www.a-ch-d.eu/MATERIALIEN/ WOERTERimKOPF/Merz-Paget-Petö_Handout.pdf .

PORTMANN-TSELIKAS, Paul (2011) Spracherwerb, grammatische Begriffe und sprachliche Phänomene. Überlegungen zu einem unübersichtlichen Lernfeld. K. Köpcke et A. Ziegler (dir.), Grammatik - Lehren, Lernen, Verstehen. Zugänge zur Grammatik des Gegenwartsdeutschen. Berlin, Boston : De Gruyter, 71-91.

RALL, Marlene (2001) Grammatikvermittlung. G. Helbig, L. Götze, G. Henrici et H.-J. Krumm (dir.), Deutsch als Fremdsprache. (Ein internationales Handbuch. 2. Halbbd). Berlin/New York : De Gruyter, 880-886.

REDDER, Angelika/Jochen REHBEIN (dir.) (1999) Grammatik und mentale Prozesse. Tübingen : Stauffenberg Verlag Brigitte Narr.

REIMANN, Daniel (2016) Nonverbale Kommunikation in mehrsprachigen Lernprozessen am Beispiel des Spanischen als dritter oder spät beginnender Fremdsprache. C. Michler et D. Reimann (dir.), Sehverstehen im Unterricht der romanischen Sprachen. Tübingen : Narr, 145-182.

ROTH, Gerhard (2009) Warum sind Lehren und Lernen so schwierig? U. Herrmann (dir.), Neurodidaktik. Weinheim et Bâle : Beltz, 58-68.

RÜCKL, Michaela/Gabriele HOLZINGER (2013) Découvrons le français. Französisch interlingual. Vienne : Verlag hpt. 
SATZINGER, Christa (2016) Mit Karambolage sehen(d) verstehen. C. Michler et D. Reimann (dir.), Sehverstehen im Fremdsprachenunterricht. Tübingen : Gunter Narr, 244-262.

SEERINGER, Juliane (2015) On bouge! Mit Bodypercussion grammatische Strukturen festigen. Der fremdsprachliche Unterricht Französisch 133, 11-14.

SOUSA, David A. (2006) How the Brain Learns. Thousand Oaks, Californie : Corwin Press.

SQUIRE, Larry (1987) Memory and brain. New York : Oxford Univ. Press.

THURMAIR, Maria (2010) Grammatikwissen und Fremdsprachenerwerb: wer, was und wozu? M. Habermann (dir.), Thema Deutsch 11, 357-371.

THURMAIR, Maria (2011) Grammatik verstehen lernen mit Hilfe von Textsorten. K. Köpcke et A. Ziegler (dir.), Grammatik - Lehren, Lernen, Verstehen. Zugänge zur Grammatik des Gegenwartsdeutschen. Berlin, Boston : De Gruyter, 411-433.

TULVING, Endel (1995) Organization of memory: Quo vadis? M. S. Gazzaniga (dir.), The cognitive Neurosciences. Cambridge : MIT Press, 839-847.

WEWETZER, Hartmut (2011) Hirnforscher empfiehlt entschlackte und reformierte Lehrpläne. ZEIT Online. En ligne, le 16/10/2019. http://www.zeit.de/gesellschaft/ schule/2011-04/schule-hirnforschung.

ZIPPEL, Wolfgang (2009) Semantik und Grammatik im Kopf. Tübingen : Günter Narr. Österreichischer Lehrplan für Lebende Fremdsprachen (Erste, Zweite). En ligne, le 16/10/2019. https://bildung.bmbwf.gv.at/schulen/unterricht/lp/ahs8_782.pdf?61ebzr.

Les RITMIMOTS, le français par l'oreille musicale. En ligne, le 16/10/2019. http://www. br-online.de/imperia/md/content/bayern/collegerad/franzoesis/3.rtf .

\section{POVZETEK}

\section{Lažjemu in bolj navdihujočemu poučevanju slovnice naproti}

Kot je znano, je bil pomen slovnice pri poučevanju tujega jezika od nekdaj najbolj priljubljena tema razprav med jezikovnimi strokovnjaki, didaktiki in jezikoslovci (Thurmair 2010: 357). Mnenje številnih sodelavcev, da je poučevanje (v našem primeru francoske) slovnice vse težje, med drugim odpira naslednja vprašanja:

- Kako lahko poučevanje jezika naredimo učinkovitejše?

- $\quad$ Ali in v kakšni obliki je poučevanje slovnice sploh še smiselno, tako da »optimizira učni proces« (Thurmair 2010: 357), pri katerem učenci pridobivajo znanje slovnice in urijo svoje veščine?

- Kako lahko učencem pomagamo pri razumevanju slovnice in pri pridobivanju slovničnih znanj, obenem pa jih motiviramo za učenje jezika? 
Iskanje odgovorov na ta vprašanja za učitelje nikakor ni enostavno, predstavlja pa zanimiv izziv. Po zadnji razpravi o rezultatih raziskave PISA in z njo povezani kritiki izobraževanja učiteljev strokovnjaki na področju didaktike in pedagoškega dela več pozornosti namenjajo raziskovanju možganov. Kljub nekaterim kritičnim odzivom je treba priznati, da so nevroznanstveniki prišli do dragocenih in vznemirljivih odkritij, ki sicer sama po sebi ne prinašajo rešitve, ob povezovanju s primernimi didaktičnimi nalogami pa lahko pripomorejo k doseganju dobrih učnih rezultatov. Da bi utemeljila to trditev, avtorica v prispevku predstavi konkretne primere, ki izhajajo iz izkušenj, ki jih je pridobila pri poučevanju francoščine.

Ključne besede: poučevanje tujega jezika, nevrodidaktika, nevroznanost, poučevanje slovnice, jezikovna zavest

\section{ABSTRACT}

\section{For an easier and more motivating use of grammar}

"As we all know, the importance of grammar in foreign language teaching ...has been the most popular object of dispute among practitioners, didacticians and linguists since forever" (Thurmair 2010: 357).

Moreover, the many comments from colleagues that it is becoming increasingly difficult to teach grammar, in our case French grammar, raise the following questions:

- how to make language teaching more effective,

- whether and in what form grammar teaching still makes sense and "can optimise acquisition processes" (Thurmair 2010: 357), through which learners acquire grammatical knowledge and skills,

- $\quad$ how to help learners understand grammar and acquire grammatical knowledge while motivating them to learn the language.

This is certainly not an easy task for teachers, but is a nice challenge!

Since the last PISA debate and the associated negative comments on the actual teacher training, brain research has been given a new status in the field of didactics and pedagogy. In spite of critical voices, it must be admitted that neuroscientists have achieved valuable and exciting research results that are not in themselves a solution to the problems outlined above, but in combination with suitable didactic exercises can achieve good learning effects.

Some concrete examples from French lessons will be used to illustrate this.

Keywords: foreign language teaching, neurodidactics, neuroscience, grammar teaching, language awareness 


\section{RÉSUMÉ}

De nombreux retours de collègues enseignants selon lesquels il devient de plus en plus difficile d'enseigner la grammaire, dans notre cas la grammaire française, soulèvent naturellement la question de savoir

- comment rendre l'enseignement des langues efficace,

- $\quad$ si l'enseignement de la grammaire a encore du sens et sous quelle forme,

- $\quad$ si on peut « optimiser les processus d'acquisition » (Thurmair 2010: 357), par lesquels les apprenants acquièrent des connaissances et des compétences grammaticales,

- comment aider les apprenants à comprendre la grammaire et à acquérir des connaissances grammaticales tout en les motivant à apprendre la langue.

Ce n'est certes pas une tâche facile pour les enseignants, mais un beau défi à relever !

Au moins depuis le dernier débat autour de PISA et la remise en question de la formation des enseignants, la recherche sur le cerveau a acquis un nouveau statut dans le domaine de la didactique et de la pédagogie. Malgré toutes les critiques, qui reviennent sans cesse, il faut admettre que les neuroscientifiques sont arrivés dans leurs travaux de recherche à des résultats précieux et passionnants, qui ne sont pas en soi une solution, mais qui, combinés à des activités didactiques appropriées, peuvent mener à une amélioration de l'enseignement et de l'apprentissage.

Nous illustrerons notre propos de quelques exemples concrets tirés de nos leçons de français.

Mots-clés : didactique des langues étrangères, neuro-didactique, neurosciences, enseignement de la grammaire, apprentissage de la langue 